\title{
Second XP Workshop about Dealing with Usability in an Agile Domain
}

\author{
Ana Maria Moreno and Agustin Yagüe \\ Universidad Politecnica de Madrid \\ Madrid, Spain \\ ammoreno@fi.upm.es, agustin.yague@upm.es
}

\begin{abstract}
The Second Workshop "Dealing with Usability in an Agile Domain" is aimed to be a forum for discussing these approximations for the intersection of agility and usability. More concretely, issues regarding the implications of usability in agile environments, alternatives for integrating HCI techniques in an agile process, approaches for incorporating usability features into agile artifacts or open issues in the integration of agile and usability where discussed.
\end{abstract}

Keywords: Agile development, usability patterns, user stories, HCI.

\section{Presentation}

The integration of usability engineering and agile software development practices is an emerging challenge. Both the agile and the HCI community have recognized the need and the difficulties to incorporate efficient usability practices in this domain. During XP 2010 a first edition of this workshop was held with about 25 attendees, who actively participated in an interesting debate about the topic and highlighted many open issues.

XP 2011 workshop is aimed to keep on being a forum for discussing approximations for the intersection of agility and usability. Participation would be open to all XP2011 attendees.

The Workshop is scheduled in two 90 minutes sessions. First sesion will be structured around an invited talk by a representative speaker in the area and the presentation and discussion of individual position papers. Second sesion will be opened to all the attendees to propose open issues to discuss about usability $\&$ agility and will be followed by a final debate about the current practice and open issues in the integration of usability and agility.

Among our learning outcomes we find contributing to understanding the implications of usability in agile environments, exploring alternatives for integrating HCI techniques in an agile proces, opening up approaches for incorporating usability requirements into agile artefacts, or identifying open issues in the integration of agile and usability. 